\title{
A PROOF OF THE POWER SERIES EXPANSION WITHOUT DIFFERENTIATION THEORY
}

\section{A. J. MACINTYRE AND W. JOHN WILBUR}

1. Introduction. Morera's theorem [8], [9] raised the suggestion that complex function theory might be based on integration rather than differentiation. This has been taken up by Heffter. The problem according to Heffter is to obtain the power series expansion from the assumption of in tegrability. Integrability here means that the contour integral $\int f(z) d z$ depends only on the end points of the curve of integration. His method is based on the "fundamental theorem" [5, pp. 45, 47].

If $f(z)$ is continuous and integrable and if $g(z)$ is differentiable, then $f(z) g(z)$ is integrable.

He then obtains Cauchy's integral representation and the power series expansion follows in the usual way.

Heffter's fundamental theorem can be improved by supposing only that $g(z)$ is integrable and satisfies a Lipschitz condition instead of assuming differentiability. The power series expansion can be obtained independently of differentiation theory and without the use of Cauchy's integral formula. These and some related results are obtained below. But a simple and direct proof that the product of two continuous integrable functions is integrable is still lacking.

The question of obtaining the power series expansion or other properties of analytic functions from differentiability without using Cauchy's integral representation has attracted more attention. For this see G. T. Whyburn, Topological analysis and the articles by E. Connell and P. Porcelli given in the references.

2. The following discussion is aimed at obtaining certain properties of integrable, continuous functions of a complex variable without introducing the concept of differentiability.

Lemma A. If $R$ is a simply connected region and $f(z)$ is a continuous function in $R$ and $\int_{\Delta} f(z) d z=0$ for every triangle $\Delta$ in $R$ then

$$
\int_{C} f(z) d z=0
$$

for every closed path $C$ in $R$.

For the proof see [6, pp. 51-53].

Received by the editors April 18, 1966. 
Lемма B. If $f(z)$ is continuous in a region $R$ and integrable in every simply connected subregion of $R$, and $C$ and $C^{\prime}$ are simple closed paths having both themselves and their interiors contained in $R \cup\{w\}$ where w is a fixed point in the intersection of the interiors of $C$ and $C^{\prime}$, then $\int_{C} f(z) d z=\int_{C^{\prime}} f(z) d z$.

Proof. Choose a circle completely contained in the intersection of the interiors of $C$ and $C^{\prime}$ with center $w$ and apply the proof of $[6$, Theorem 1, p. 56].

THEOREM A. If $f(z)$ and $g(z)$ are continuous integrable functions of $z$ in a simply connected region $R$ and $f(z)$ satisfies a Lipschitz condition at each point of $R$, then $f(z) g(z)$ is integrable in $R$.

Proof. Let $\Delta_{1}$ be a triangle in $R$ and consider $\int_{\Delta_{1}} f(z) g(z) d z$. We write $A=\left|\int_{\Delta_{1}} f(z) g(z) d z\right|$. Next, beginning with $\Delta_{1}$, we inductively break $\Delta_{n}$ in to four congruent triangles each similar to $\Delta_{n}$ and from among them choose

$$
\Delta_{n+1} \ni\left|\int_{\Delta_{n}} f(z) g(z) d z\right| \leqq 4\left|\int_{\Delta_{n+1}} f(z) g(z) d z\right| .
$$

$\mathrm{By}$ the theorem on nested continua there is one and only one point $z_{0}$ in all $\Delta_{n}$.

Since $f(z)$ satisfies a Lipschitz condition at $z_{0}$ there exists a constant $M$ and a neighborhood $N_{\delta}$ of $z_{0} \ni\left|f(z)-f\left(z_{0}\right)\right| \leqq M\left|z-z_{0}\right|$, $\forall z \ni\left|z-z_{0}\right| \leqq \delta$. Since $g(z)$ is continuous at $z_{0}$ there exists for a fixed $\epsilon>0$ a neighborhood $N_{\delta^{\prime}}$ of $z_{0} \ni\left|g(z)-g\left(z_{0}\right)\right|<\epsilon \forall z \ni\left|z-z_{0}\right| \leqq \delta^{\prime}$. We now choose $n^{\prime}$ large enough so that $\Delta_{n^{\prime}} \subset N_{\delta} \cap N_{\delta^{\prime}}$. Noting that $f(z) g\left(z_{0}\right), f\left(z_{0}\right) g(z)$, and $f\left(z_{0}\right) g\left(z_{0}\right)$ are all in tegrable functions of $z$ in $R$, it is evident that

$$
\int_{\Delta_{n^{\prime}}}\left(f(z)-f\left(z_{0}\right)\right)\left(g(z)-g\left(z_{0}\right)\right) d z=\int_{\Delta_{n^{\prime}}} f(z) g(z) d z .
$$

It follows that

$$
\begin{aligned}
A \leqq 4^{n^{\prime}}\left|\int_{\Delta_{n^{\prime}}} f(z) g(z) d z\right| & =4^{n^{\prime}}\left|\int_{\Delta_{n^{\prime}}}\left(f(z)-f\left(z_{0}\right)\right)\left(g(z)-g\left(z_{0}\right)\right) d z\right| \\
& \leqq 4^{n^{\prime}} \int_{\Delta_{n^{\prime}}}\left|f(z)-f\left(z_{0}\right)\right|\left|g(z)-g\left(z_{0}\right)\right||d z| \\
& \leqq 4^{n^{\prime}} M \frac{s_{n^{\prime}}}{2} \epsilon s_{n^{\prime}}=M \frac{s^{2}}{2} \epsilon
\end{aligned}
$$


where $s_{n^{\prime}}$ is the perimeter of $\Delta_{n^{\prime}}$ and $s$ is the perimeter of $\Delta_{1}$. We conclude that $A=0$ and $\int_{\Delta_{1}} f(z) g(z) d z=0$. Lemma A now implies the integrability of $f(z) g(z)$ in $R$.

ThEOREM B. Let $f(z)$ be integrable in a simply connected region $R$. Further let $f(z)$ satisfy a Lipschitz condition at each point of $R$ and have no zeros in $R$. If $h(z)$ is defined, continuous and integrable in $R$ then the function $g(z)=h(z) / f(z)$ is integrable in $R$.

Proof. As in the proof of Theorem A we obtain a sequence of triangles and a point $z_{0}$ in (or on) every triangle of the sequence. We then note that

$$
\int_{\Delta}\left(f(z)-f\left(z_{0}\right)\right)\left(g(z)-g\left(z_{0}\right)\right) d z=-f\left(z_{0}\right) \int_{\Delta} g(z) d z .
$$

THEOREM C. If $f(z)$ is continuous and integrable in a simply connected region $R$, and if $z_{0}$ is a fixed point in $R$, then the function

$$
F(z)=\int_{z_{0}}^{z} f(\zeta) d \zeta
$$

is also integrable in $R$.

Proof. We need only consider $\int_{z_{0}}^{z} F(\zeta) d \zeta$ along polygonal paths. For iterated integrals with real variables we have the formula

$$
\int_{0}^{1}\left\{\int_{0}^{t} g(u) d u\right\} d t=\int_{0}^{1}(1-t) g(t) d t .
$$

We base our discussion on an extension of this formula to the complex integral. We suppose that in $\int_{z_{0}}^{2} F(\zeta) d \zeta$ the function $F(\zeta)$ is defined by integration of $f(\zeta)$ along part of the same curve.

With a suitable parametric representation

$$
\begin{aligned}
\int_{z_{0}}^{z} F(\zeta) d \zeta & =\int_{0}^{1}\left\{\int_{0}^{t} f[w(u)] w^{\prime}(u) d u\right\} w^{\prime}(t) d t \\
& =\int_{0}^{1}\left\{\int_{u}^{1} f[w(u)] w^{\prime}(u) w^{\prime}(t) d t\right\} d u \\
& =\int_{0}^{1}[w(1)-w(u)] f[w(u)] w^{\prime}(u) d u \\
& =\int_{z_{0}}^{2}[z-\zeta] f(\zeta) d \zeta .
\end{aligned}
$$


This last integral is independent of the path of integration by Theorem $\mathrm{A}$ and $F(z)$ is in tegrable.

Lemma C. If $C_{r}$ stands for a circle of radius $r$ and center $z_{0}$ and $f(z)$ is a function continuous in a region $R$ containing $z_{0}$, then

$$
\lim _{r \rightarrow 0} \int_{C_{r}} \frac{f(z)}{z-z_{0}} d z=2 \pi i f\left(z_{0}\right) .
$$

The proof of this simple lemma is omitted.

Lemma D. If $C$ is a circle with center $z_{0}$ and $f$ is a function continuous on the boundary of $C$ and if for all $n \geqq 1, \int_{C}\left(f(z) /\left(z-z_{0}\right)^{n}\right) d z=0$, then for all $z_{1}$ in the interior of $C, \int_{C}\left(f(z) /\left(z-z_{1}\right)\right) d z=0$.

Proof. Let $z_{1}$ be in the interior of $C$. Then

$$
\begin{aligned}
\int_{C} \frac{f(z)}{z-z_{1}} d z & =\int_{C} f(z)\left\{\frac{1}{\left(z-z_{0}\right)-\left(z_{1}-z_{0}\right)}\right\} d z \\
& =\int_{C} f(z)\left\{\sum_{n=0}^{\infty} \frac{\left(z_{1}-z_{0}\right)^{n}}{\left(z-z_{0}\right)^{n+1}}\right\} d z \\
& =\int_{C}\left\{\sum_{n=0}^{\infty} f(z) \frac{\left(z_{1}-z_{0}\right)^{n}}{\left(z-z_{0}\right)^{n+1}}\right\} d z .
\end{aligned}
$$

Since $f(z)$ is continuous it has a finite maximum modulus on $C$ and the series under the integral sign is uniformly convergent on $C$. We may thus integrate term by term obtaining

$$
\begin{aligned}
\int_{C} \frac{f(z)}{z-z_{1}} d z & =\sum_{n=0}^{\infty}\left(z_{1}-z_{0}\right)^{n} \int_{C} \frac{f(z)}{\left(z-z_{0}\right)^{n+1}} d z \\
& =\sum_{n=0}^{\infty}\left(z_{1}-z_{0}\right)^{n} \cdot 0=0 .
\end{aligned}
$$

TheOREM D. If $f$ is an integrable, continuous function in a simply connected region $R$ and $C$ is a circle in $R$ with center $z_{0}$, then for all $z$ in the interior of $C$ we have

$$
f(z)=\sum_{n=0}^{\infty}\left\{\frac{1}{2 \pi i} \int_{c} \frac{f(\zeta) d \zeta}{\left(\zeta-z_{0}\right)^{n+1}}\right\}\left(z-z_{0}\right)^{n} .
$$

Proof. Let the radius of $C$ be $r$ and let

$$
g(z)=\sum_{n=0}^{\infty}\left\{\frac{1}{2 \pi i} \int_{C} \frac{f(\zeta) d \zeta}{\left(\zeta-z_{0}\right)^{n+1}}\right\}\left(z-z_{0}\right)^{n} .
$$


We calculate a lower bound for the radius of convergence of the series for $g(z)$. Let $M$ be a positive upper bound for the modulus of $f(z)$ on $C$ and let $\rho$ stand for the radius of convergence of the series. Then

$$
\begin{aligned}
\frac{1}{\rho} & =\limsup _{n}\left(\left|\frac{1}{2 \pi i} \int_{C} \frac{f(\zeta) d \zeta}{\left(\zeta-z_{0}\right)^{n+1}}\right|\right)^{1 / n} \\
& \leqq \limsup _{n}\left(\frac{1}{2 \pi} \int_{C} \frac{M|d \zeta|}{\left|\zeta-z_{0}\right|^{n+1}}\right)^{1 / n} \\
& =\limsup _{n}\left(\frac{1}{2 \pi} \frac{M 2 \pi r}{r^{n+1}}\right)^{1 / n}=\limsup _{n} \frac{M^{1 / n}}{r}=\frac{1}{r} .
\end{aligned}
$$

Thus $\rho \geqq r$.

Now let $z_{1}$ be in the interior of $C$ and let $C^{\prime}$ be a circle smaller than but concentric with $C$ also having $z_{1}$ in its interior. Then we calculate $\int_{C^{\prime}}\left((f(z)-g(z)) /\left(z-z_{0}\right)^{m}\right) d z$ for $m \geqq 1$. We have

$$
\int_{C^{\prime}} \frac{f(z)-g(z)}{\left(z-z_{0}\right)^{m}} d z=\int_{C^{\prime}} \frac{f(z)}{\left(z-z_{0}\right)^{m}} d z-\int_{C^{\prime}} \frac{g(z)}{\left(z-z_{0}\right)^{m}} d z
$$

and since the series for $g(z)$ is uniformly convergent on $C^{\prime}$ we may integrate term by term obtaining

$$
\begin{aligned}
\int_{C^{\prime}} \frac{g(z)}{\left(z-z_{0}\right)^{m}} d z & =\sum_{n=0}^{\infty}\left\{\frac{1}{2 \pi i} \int_{C} \frac{f(\zeta)}{\left(\zeta-z_{0}\right)^{n+1}} d \zeta\right\} \int_{C^{\prime}}\left(z-z_{0}\right)^{n-m} d z \\
& =\int_{C} \frac{f(\zeta)}{\left(\zeta-z_{0}\right)^{m}} d \zeta .
\end{aligned}
$$

It follows that

$$
\int_{C^{\prime}} \frac{f(z)-g(z)}{\left(z-z_{0}\right)^{m}} d z=\int_{C^{\prime}} \frac{f(z)}{\left(z-z_{0}\right)^{m}} d z-\int_{C} \frac{f(z)}{\left(z-z_{0}\right)^{m}} d z .
$$

But by a simple application of Theorems $\mathrm{A}$ and $\mathrm{B}, f(z) /\left(z-z_{0}\right)^{m}$ is integrable in every simply connected subregion of $R$ not containing $z_{0}$ and thus by Lemma B,

$$
\int_{C^{\prime}} \frac{f(z)}{\left(z-z_{0}\right)^{m}} d z=\int_{C} \frac{f(z)}{\left(z-z_{0}\right)^{m}} d z .
$$

Consequently for $m \geqq 1$,

$$
\int_{C^{\prime}} \frac{[f(z)-g(z)]}{\left(z-z_{0}\right)^{m}} d z=0
$$


and by Lemma $\mathrm{D}$,

$$
\int_{C^{\prime}} \frac{[f(z)-g(z)]}{\left(z-z_{1}\right)} d z=0 .
$$

Now $g(z)$ is a power series and so certainly integrable in the interior of $C$. We thus have $f(z)-g(z)$ integrable in the interior of $C$ and by Theorem $\mathrm{B},(f(z)-g(z)) /\left(z-z_{1}\right)$ integrable in every simply connected subregion of the interior of $C$ not containing $z_{1}$. It follows by Lemma $B$ that we obtain

$$
0=\int_{C^{\prime}} \frac{[f(z)-g(z)]}{\left(z-z_{1}\right)} d z=\int_{C_{r}} \frac{[f(z)-g(z)]}{\left(z-z_{1}\right)} d z
$$

for all sufficiently small $r$, where $C_{r}$ is a circle of radius $r$ and center $z_{1}$. Now by Lemma $C$ we have $2 \pi i\left[f\left(z_{1}\right)-g\left(z_{1}\right)\right]=0$ or $f\left(z_{1}\right)=g\left(z_{1}\right)$.

We note in conclusion that both the Cauchy integral representation and Morera's theorem are corollaries of Theorem D.

\section{REFERENCES}

1. E. H. Connell, A classical theorem in complex variables, Amer. Math. Monthly 72 (1965), 729-732.

2. E. H. Connell and P. Porcelli, An algorithm of J. Schur and the Taylor series, Proc. Amer. Math. Soc. 13 (1962), 232-235.

3. P. Porcelli and E. H. Connell, $A$ proof of the power series expansion without Cauchy's formula, Bull. Amer. Math. Soc. 67 (1961), 177-181.

4. L. Heffter, Kurvenintegrale und Begründung der Funktionentheorie, Springer, Heidelberg, 1948.

5. - Begründung der Funktionentheorie auf alten und neuen Wegen, Springer, Berlin, 1955.

6. K. Knopp, Theory of functions, Part I, Dover, New York, 1945.

7. A. J. Macintyre, $A$ direct proof of Morera's theorem, Arch. Math. 8 (1957), 374-375.

8. G. Morera, Un teorema fundamentale nella teoria delle funzioni di una variabile complessa, Rend. Res. Ist. Lombardia (2) 19 (1886).

9. - Sulla definizione di funzione di una variabile complessa, Atti di Torino 37 (1902), 99-102.

10. G. T. Whyburn, Topological analysis, Princeton Univ. Press, Princeton, N. J., 1958.

11. - Developments in topological analysis, Fund. Math. 50 (1961-1962), 305-318.

University of California, Davis 